\title{
MONOCLONAL GAMMOPATHY OF UNDETERMINED SIGNIFICANCE CLINIC DURING THE COVID-19 PANDEMIC: CARING FOR THE VULNERABLE IN AN ACADEMIC MEDICAL CENTER
}

\author{
Holly Lee ${ }^{1}$, Jason Tay ${ }^{1,2}$, Lesley Street ${ }^{1}$, Peter Duggan ${ }^{1,2}$, and Victor H. Jiménez-Zepeda ${ }^{1,2 *}$ \\ ${ }^{1}$ Department of Medical Oncology and Hematology, Tom Baker Cancer Centre, Calgary; ${ }^{2}$ Charbonneau Cancer \\ Research Institute, Calgary, Canada
}

\begin{abstract}
Background: Patients with monoclonal gammopathy of undetermined significance (MGUS) have clinical features including older age, presence of medical comorbidities, susceptibility to infections, and thrombotic tendencies which are relevant when assessing their risk during the coronavirus disease (COVID-19) pandemic. Objective: To study the vulnerability of patients with MGUS during the COVID-19 pandemic, we assessed the local management of MGUS patients and their clinical outcomes. Methods: Retrospective chart reviews were performed for all patients with MGUS seen at a university medical center clinic (2014-2020). Results: A total of 228 MGUS patients were included; 211 patients are alive, 7 patients died before the pandemic, and 10 patients died since the pandemic declaration. The mean age and the overall survival (OS) of the patients who died before versus during the pandemic were 83.0 versus 75.2 years, $p=0.4$, and OS 40.6 versus 53.2 months, $p=0.3$, respectively. One patient died of COVID-19. Nine patients had venous thromboembolisms (VTE), all of which occurred before the pandemic onset. Conclusions: There were no significant differences found in the mean age or OS of the MGUS patients who died before versus after the pandemic onset. An increase in VTE rates was not seen. Study results are limited by small patient numbers. (REV INVEST CLIN. 2021;73(4):259-64)
\end{abstract}

Key words: Monoclonal gammopathy of undetermined significance. Coronavirus disease. Clinical outcome.

\section{INTRODUCTION}

The coronavirus disease 2019 (COVID-19), caused by severe acute respiratory syndrome coronavirus-2 (SARS-CoV-2), is a significant global health challenge with over 83 million confirmed cases as of December 2020. The World Health Organization (WHO) has declared the COVID-19 pandemic on March 11, $2020^{1}$. COVID-19 is associated with a wide range of clinical syndromes, including respiratory, cardiac,
*Corresponding author:

Víctor H. Jiménez-Zepeda

E-mail: Victor.Zepeda@albertahealthservices.ca
Received for publication: 04-02-2021

Approved for publication: 20-04-2021

DOI: $10.24875 / R I C .21000078$

0034-8376 / (c) 2021 Revista de Investigación Clínica. Published by Permanyer. This is an open access article under the CC BY-NC-ND license (http://creativecommons.org/licenses/by-nc-nd/4.0/). 
thrombotic, and inflammatory complications ${ }^{2-4}$, and it is essential to identify patients who are at high risk of these morbidities and poor outcomes.

Monoclonal gammopathy of undetermined significance (MGUS) is a premalignant plasma cell disorder occurring in approximately $5 \%$ of patients over the age of $70^{5}$. A small proportion of MGUS patients progresses to multiple myeloma, lymphoma, or AL amyloidosis (risk of progression of $1 \%$ /year5). While MGUS is otherwise considered a generally "benign" condition, MGUS patients have several clinical features that are relevant to consider when assessing their risk during the COVID-19 outbreak.

In addition to their older median age ( 72 years ${ }^{5}$ ) and medical comorbidities ${ }^{6}$, MGUS patients have previously been shown to have increased susceptibility to infections partly related to immunoparesis ${ }^{7,8}$, greater incidences of thrombotic events compared to the general population ${ }^{9,10}$, and higher mortality compared to expected age-matched controls 5 . Given these factors, it is important to study the vulnerability of MGUS patients to COVID-19 infection and its complications; therefore, we assessed our local experience of MGUS patient outcomes during this pandemic.

\section{METHODS}

Retrospective chart reviews of all consecutive patients with MGUS seen at the University of Calgary Medical Clinics (an academic medical center) between 2014 and 2020 were completed. Patients were managed according to local institution guidelines. This project has been approved by the Health Research Ethics Board.

\section{Statistical analysis}

All statistical analyses were performed using the SPSS 24.0 software. Independent samples t-test was used to compare mean values (Table 1 ). Kaplan-Meier survival curve analysis was performed for the assessment of median survival and for the generation of figure 1 .

\section{RESULTS}

A total of 228 patients with MGUS followed at the University of Calgary Medical Clinic were assessed.
Clinical characteristics are reported in table 1. Median age of the group was 73 years. Since the beginning of the pandemic, all MGUS cases have been followed up by virtual appointments; 190 patients have been assessed through telemedicine.

At the time of analysis, 211 patients are alive, 7 patients died before the start of the pandemic, and 10 patients died since the pandemic declaration by the WHO on March 11, 2020. Three patients died in 2019 compared to 10 since the COVID-19 pandemic declaration, suggesting an excess of deaths in the year 2020. The median overall survival (OS) for the 228 patients was not reached (Fig. 1). Median followup was 55 months. There were no significant differences between the mean age and the OS of the patients who died before versus during the pandemic (age 83.0 vs. 75.2 years, $p=0.4$, OS 40.6 vs. 53.2 months, $p=0.3$ ) (Table 1 ).

Of the 10 patients who died during the pandemic, one patient died of COVID-19 a week after the diagnosis. Among the remaining nine MGUS patients, four patients had COVID-19 testing which was negative. One patient died of complications with viscous perforation with gastrointestinal (GI) involvement of $A L$ amyloidosis, two patients died of an exacerbation of chronic cardiac comorbidities, and six patients died of undocumented or unknown causes.

At the onset of the pandemic in the beginning of year 2020, patients were eligible to have COVID-19 testing done if they had fulfilled the criteria for testing in our province which included the presence of fevers, new or worsening cough, dyspnea, rhinorrhea, chills, sore throat, painful swallowing, headache, muscle or joint ache, malaise, fatigue, severe exhaustion, nausea, vomiting, diarrhea, unexplained loss of appetite, loss of sense of smell or taste, or conjunctivitis. From the end of May 2020 to November 2020, testing criteria were expanded such that anyone was eligible for COVID-19 testing regardless of symptoms. Testing was done by nucleic acid amplification using primers targeting the $E$ (envelope protein) gene of SARS-CoV-2. In the general population of 4.4 million in the province of Alberta, $1,650,655$ people had been tested, 100,428 cases were positive, and 1046 patients died as of December $30,2020^{11}$. In the 228 MGUS clinic population, 85 patients were tested (37.3\%). Three patients so 
Table 1. MGUS clinic patient characteristics

\begin{tabular}{|c|c|c|c|c|}
\hline & $\begin{array}{l}\text { Entire MGUS } \\
\text { cohort } \\
(n=228) \\
\text { Median }\end{array}$ & $\begin{array}{l}\text { Patients who died } \\
\text { before the pandemic } \\
\left(\begin{array}{l}(n=7) \\
\text { Mean }\end{array}\right.\end{array}$ & $\begin{array}{l}\text { Patients who died } \\
\text { during the pandemic } \\
(\mathrm{n}=10) \\
\text { Mean }\end{array}$ & $\begin{array}{l}\text { Independent } \\
\text { sample t-test } \\
\quad \text { p-value }\end{array}$ \\
\hline Age & 73 & 83.0 & 75.2 & 0.4 \\
\hline Male & $128(56 \%)$ & 6 & 7 & 0.1 \\
\hline Overall survival (months) & Not reached & 40.6 & 53.2 & 0.3 \\
\hline Hemoglobin & 136 & 143 & 138.5 & 0.9 \\
\hline Lymphocytes & 1.6 & 1.7 & 1.0 & 0.9 \\
\hline Creatinine & 90 & 138.5 & 124 & 0.4 \\
\hline Serum $M$ protein $(g / L)$ & 3.5 & 5.84 & 5 & 0.2 \\
\hline Bone marrow plasma cells $(n=153)$ & $3 \%$ & $5(n=4)$ & $4.5(n=5)$ & 0.1 \\
\hline NT-proBNP & 124 & 4751 & 696 & 0.01 \\
\hline \multicolumn{5}{|l|}{ Frequency } \\
\hline IgG MGUS & $115(50.5 \%)$ & $2(28.6 \%)$ & $6(60.0 \%)$ & \\
\hline IgA MGUS & $20(8.7 \%)$ & $1(14.3 \%)$ & $2(20 \%)$ & \\
\hline IgM MGUS & $35(15.3 \%)$ & $1(14.3 \%)$ & $1(14.3 \%)$ & \\
\hline Biclonal MGUS & $8(3.5 \%)$ & 0 & 0 & \\
\hline Light chain MGUS & $44(19.3 \%)$ & $3(43.0 \%)$ & 0 & \\
\hline Heavy chain and light chain MGUS & $6(2.6 \%)$ & 0 & $1(10 \%)$ & \\
\hline \multicolumn{5}{|l|}{ MGUS risk } \\
\hline Low & $56(24.6 \%)$ & 0 & $4(40 \%)$ & \\
\hline Low-intermediate & $83(36.4 \%)$ & $1(14.3 \%)$ & $4(40 \%)$ & \\
\hline High-intermediate & $30(13.2 \%)$ & $2(28.6 \%)$ & $1(10 \%)$ & \\
\hline High & $2(0.9 \%)$ & 0 & 0 & \\
\hline Light chain MGUS & $44(19.3 \%)$ & $3(43.0 \%)$ & 0 & \\
\hline Biclonal MGUS & $8(3.5 \%)$ & 0 & 0 & \\
\hline Missing data & $5(2.2 \%)$ & $1(14.3 \%)$ & $1(10 \%)$ & \\
\hline \multicolumn{5}{|l|}{ Immunoparesis } \\
\hline None & $146(64 \%)$ & $3(42.9 \%)$ & $6(60 \%)$ & \\
\hline One immunoglobulin decreased & $45(19.7 \%)$ & $1(14.3 \%)$ & $3(30 \%)$ & \\
\hline Two immunoglobulins decreased & $25(11.0 \%)$ & $3(42.9 \%)$ & $1(10 \%)$ & \\
\hline Three immunoglobulins decreased & $1(0.4 \%)$ & 0 & 0 & \\
\hline Missing data & $11(4.8 \%)$ & 0 & 0 & \\
\hline \multicolumn{5}{|l|}{ Previous medical comorbidities } \\
\hline $\begin{array}{l}\text { Previous venous thromboembolism } \\
\text { (DVT/PE) }\end{array}$ & 9 & 0 & 2 & \\
\hline Atrial fibrillation & 33 & 4 & 3 & \\
\hline Hypertension & 97 & 2 & 5 & \\
\hline Diabetes & 52 & 0 & 3 & \\
\hline \multicolumn{5}{|c|}{$\begin{array}{l}\text { (includes psoriasis, rheumatoid arthritis, rheumatic polymyalgia, chronic inflammatory demyelinating polyneuropathy, } \\
\text { hypothyroidism, Crohn's disease, multiple sclerosis, microscopic colitis, systemic lupus erythematosus, Sjogren's syndrome, } \\
\text { GAD65 positive Stiff syndrome, pernicious anemia, anti-MAG antibody, Behcet's disease, } \\
\text { and urticarial vasculitis) }\end{array}$} \\
\hline
\end{tabular}

MGUS: monoclonal gammopathy of undetermined significance. 
Figure 1. Overall survival (OS) of monoclonal gammopathy of undetermined significance clinic patients in months $(n=228)$. Cumulative survival (Cum survival), OS months.

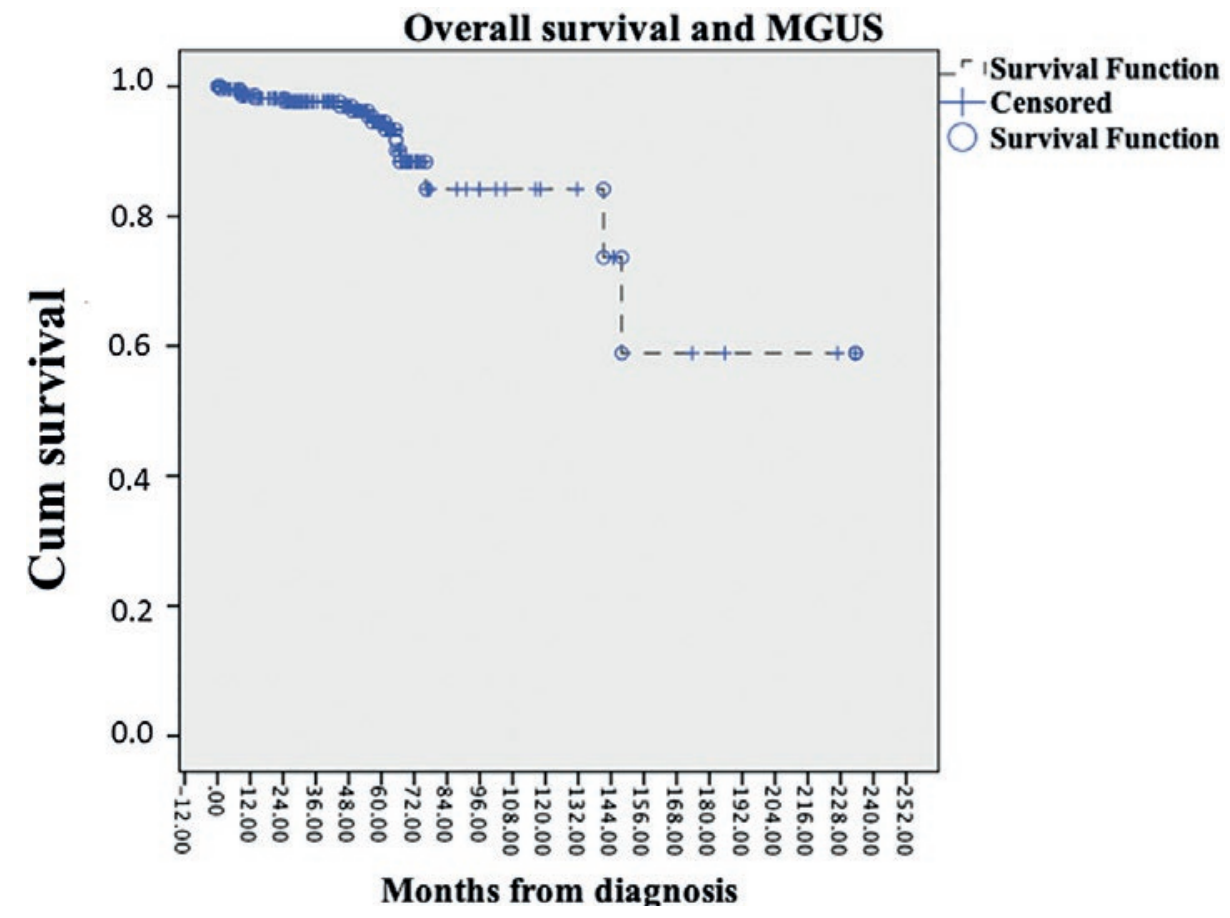

far have tested positive for SARS-CoV-2; one patient died of COVID-19 and the other two are alive at the time of submission. The incidence of positive cases among our MGUS patient cohort is too small to understand the impact of the expanded local COVID-19 testing eligibility which included testing asymptomatic patients.

The one patient with MGUS who died of COVID-19 a week after testing positive was a male in his late $80 \mathrm{~s}$ whose comorbidities included advanced dementia and hypertension. He did not have immunoparesis. The other two patients with MGUS and COVID-19 were a female in her 70s with hypertension, hyperparathyroidism, without immunoparesis, and a male in his 30 s with end-stage renal disease, obesity, hypertension, and hypothyroidism, who had immunoparesis involving lgG.

In the MGUS cohort, five patients have progressed; two patients developed lymphoma, one patient developed smoldering myeloma, one patient developed $\mathrm{AL}$ amyloidosis and symptomatic multiple myeloma, and one patient progressed to AL amyloidosis with
Gl involvement (duodenal and gastric). All progression events occurred before the pandemic onset, except for the one patient who developed AL amyloidosis with $\mathrm{Gl}$ involvement. This patient had a complication of viscous perforation and died. Median time to progression in the five patients was 44.6 months. Six patients deemed to have monoclonal gammopathy of clinical significance (MGCS) were transferred to the cancer center to expedite required investigations.

Nine patients had venous thromboembolisms (VTE), and all VTE events occurred before the pandemic onset (Table 1). One patient had a pulmonary embolism $(\mathrm{PE})$, seven patients had deep vein thrombosis (DVT), and one patient had both a PE and a DVT.

Immunoparesis was found in 71 (31.1\%) out of 228 patients. Forty-five patients $(19.7 \%)$ had one affected immunoglobulin, 25 patients (11\%) had two affected immunoglobulins, and 1 patient $(0.4 \%)$ had decreased levels of three immunoglobulins. Data on immunoglobulin levels at diagnosis were missing in 11 patients. 


\section{DISCUSSION}

The province of Alberta has declared a public health emergency as it undergoes a recurrent rapid surge in COVID-19 infection rates with the latest reported active cases of 14,555 as of January 2021 since the nadir of 339 active cases back in June 2020. The number of active cases continues to rise with over 1000 new cases diagnosed daily. In the small MGUS clinic population presented here, COVID-19 testing has been done in $37.3 \%$ of the patients ( 85 out of 228 patients) compared with the testing rate of $37.5 \%$ in the general population within the province (1,650,655 people out of approximately 4.4 million). The incidence rate of COVID-19 in the MGUS clinic is $1.3 \%$ ( 3 out of 228 patients), compared with the rate in the general population within the province which stands at around $2.28 \%(100,428$ out of 4.4 million). Unfortunately, the incidence rate in the general Alberta population continues to increase at the time of this writing (January 2021).

To assess the true susceptibility of MGUS patients to COVID-19, consistent testing is essential. It is possible that many MGUS patients are asymptomatic and well. Potentially, a number of MGUS patients may be reluctant to seek medical attention ${ }^{12}$ due to fear of COVID-19 despite developing new symptoms. With their medical complexity, older age, and frailty, patients may be offered comfort care without COVID-19 screening when severe illnesses occur.

With small patient numbers, our findings are inconclusive as to whether the older age, presence of comorbidities, inherent susceptibility to infections, and thrombotic tendencies of MGUS patients ${ }^{7,10}$ truly confer additional risk for poor outcomes during this pandemic. There were no significant differences found in the mean age, OS, or in the selected biochemical parameters (Table 1) of MGUS patients who died before or after the pandemic onset. Patient numbers were small with low incidences of COVID-19 to assess other endpoints such as intensive care unit (ICU) admission or ventilator support requirements.

Neither the MGUS risk score (based on one point for each of non-lgG monoclonal protein, abnormal free light chain ratio, and serum monoclonal protein $\geq$ ro $\mathrm{g} / \mathrm{L}^{13}$ ) nor immunoparesis appeared to be associated with death in our patient cohort. While MGUS patients carry a higher risk of thrombosis than the general population, an increase in VTE events was not seen since the pandemic onset.

Of seven MGUS patients with COVID-19 showed that despite having high-risk comorbidities, none of the patients required ICU care, and six of the seven patients recovered from the infection. A recent case series $^{14}$ of 7 MGUS patients with COVID-19 showed that despite having high risk comorbidities, none of the patients required ICU care, and 6 of the $7 \mathrm{pa}$ tients recovered from the infection. Similarly, in a report of 127 patients with plasma cell disorders who had COVID-19, including 20 MGUS patients ${ }^{15}$, their older age ( $>65$ ) or hypogammaglobulinemia was not associated with death or ICU admissions.

Overall, our study with a small patient number did not identify increased COVID-19-related risk in MGUS patients. In future studies, it would be important to assess whether COVID-19 infection affects the rate of progression from MGUS to symptomatic disease secondary to asymptomatic inflammatory states contributing to disease progression. Larger population-based studies with longer followup periods are required to understand the full impact of COVID-19 on the MGUS patients' outcomes. Furthermore, as per the latest International Myeloma Working Group recommendations, all patients with MGUS are encouraged to receive vaccinations against COVID-19. Ongoing data collection will be essential to assess MGUS patients' immunological response to vaccination.

\section{REFERENCES}

1. WHO Coronavirus Disease (COVID-19) Dashboard; 2020. Available from: https://www.covid19.who.int. [Last accessed on 2021 Jan 04].

2. Wang D, Hu B, Hu C, Zhu F, Liu X, Zhang J, et al. Clinical characteristics of 138 hospitalized patients with 2019 novel Coronavirus-infected pneumonia in Wuhan, China. JAMA. 2020;323: 1061-9.

3. Spyropoulos AC, Levy JH, Ageno W, Connors JM, Hunt BJ, Iba T, et al. Scientific and standardization committee communication: clinical guidance on the diagnosis, prevention, and treatment of venous thromboembolism in hospitalized patients with COVID-19. J Thromb Haemost. 2020;18:1859-65.

4. Mehta P, McAuley DF, Brown M, Sanchez E, Tattersall RS, Manson J, et al. Monoclonal gammopathy of undetermined significance and risk of infections: a population-based study. Haematologica. 2012;97:854-8.

5. Kyle RA, Larson DR, Therneau TM, Dispenzieri A, Kumar S, Cerhan JR, et al. Long-Term Follow-up of Monoclonal Gammopathy of Undetermined Significance. N Engl J Med. 2018;378:241-9. 
6. Atkin C, Richter A, Sapey E. What is the significance of monoclonal gammopathy of undetermined significance? Clinical Medicine. 2018;18:391-6.

7. Kristinsson SY, Tang M, Pfeiffer RM, Björkholm M, Goldin LR, Blimark C, et al. Monoclonal gammopathy of undetermined significance and risk of infections: a population-based study. Haematologica. 2012;97:854-8.

8. Cherry BM, Costello R, Zingone A, Burris J, Korde N, Manasanch $\mathrm{E}$, et al. Immunoparesis and monoclonal gammopathy of undetermined significance are disassociated in advanced age. Am J Hematol. 2013;88:89-92.

9. Kristinsson SY, Fears TR, Gridley G, Turesson I, Mellqvist UH, Björkholm $M$, et al. Deep vein thrombosis after monoclonal gammopathy of undetermined significance and multiple myeloma. Blood. 2008;112:3582-6.

10. Kristinsson SY, Pfeiffer RM, Björkholm M, Goldin LR, Schulman $\mathrm{S}$, Blimark C, et al. Arterial and venous thrombosis in monoclonal gammopathy of undetermined significance and multiple myeloma: a population-based study. Blood. 2010;115:4991-8.
11. COVID-19 Alberta Statistics; 2020. Available from: https:// www.alberta.ca/stats/covid-19-alberta-statistics.htm. [Last accessed on 2021 Jan 04].

12. Molica M, Mazzone C, Cordone I, Pasquale A, Niscola P, de Fabritiis $P$. SARS-CoV-2 infection anxieties and general population restrictions delay diagnosis and treatment of acute haematological malignancies. Br J Haematol. 2020;190:e5-8.

13. Rajkumar SV, Kyle RA, Therneau TM, Melton LJ 3rd, Bradwell AR, Clark RJ, et al. Serum free light chain ratio is an independent risk factor for progression in monoclonal gammopathy of undetermined significance. Blood. 2005;106:812-7.

14. Gonzalez-Lugo JD, Bachier-Rodriguez L, Goldfinger M, Shastri A, Sica RA, Gritsman K, et al. A case series of monoclonal gammopathy of undetermined significance and COVID-19. Br J Haematol. 2020;190:e130-3.

15. Hultcrantz M, Richter J, Rosenbaum C, Patel D, Smith E, Korde $\mathrm{N}$, et al. COVID-19 Infections and Outcomes in Patients with Multiple Myeloma in New York City: a Cohort Study from Five Academic Centers, medRxiv; 2020. 